\title{
Vectorial variational inequalities on Hadamard manifolds: A tool to achieve efficient approximate solutions
}

\author{
G. Ruiz-Garzón • R. Osuna-Gómez • \\ A. Rufián-Lizana • B. Hernández-Jiménez
}

\begin{abstract}
This article has two objectives. Firstly, we will use the vector variational-like inequalities problems to achieve local approximate (weakly) efficient solutions of Vector Optimization Problem within the novel field of the Hadamard manifolds. Previously, we will introduce the concepts of generalized approximate geodesic convex functions and illustrate them with examples. We will see the minimum requirements under which critical points, solutions of Stampacchia and Minty weak variational-like inequalities and local approximate weakly efficient solutions can be identified, extending previous results from the literature for linear Euclidean spaces. Secondly, we will show an economical application, using again solutions of the variational problems to identify with Stackelberg equilibrium points on Hadamard manifolds and under geodesic convexity assumptions.
\end{abstract}

Keywords Generalized convexity · Hadamard manifold · Approximate efficient solution · Stackelberg equilibrium point

G. Ruiz-Garzón

Instituto de Desarrollo Social y Sostenible (INDESS), Universidad de Cádiz, Campus de Jerez de la Frontera, Avda. de la Universidad s/n, 11405, Jerez de la Frontera, Cádiz, Spain. E-mail: gabriel.ruiz@uca.es

R. Osuna-Gómez

Departamento de Estadística e I.O., Universidad de Sevilla, Sevilla, Spain.

E-mail: rafaela@us.es

A. Rufián-Lizana

Departamento de Estadística e I.O., Universidad de Sevilla, Sevilla, Spain.

E-mail: rufian@us.es

B. Hernández-Jiménez

Departamento de Economía, Métodos Cuantitativos e Historia Económica, Universidad Pablo Olavide, Sevilla, Spain. E-mail: mbherjim@upo.es 


\section{Introduction}

The American statistician John W. Tukey said: "It was better to have an approximate answer to the right question than an exact answer to the approximate question". This article is about Pareto's approximate solutions for the vector optimization problem on Hadamard manifolds.

Sometimes, in practice, the best idea is not to get solutions to vector optimization problems by solving them directly but through other problems that are related to the first ones. The variational problems carry out this intermediate work that this article deals in a novel context such as the Hadamard manifolds. Similarly, sometimes we also have to be satisfied with finding approximate solutions to the vector optimization problem instead of the exact solutions. Those feasible points whose objective values are at a small $\epsilon$ distance from the optimal objective vector values are considered an approximate Pareto solution. In some particular problems, the limit of approximate Pareto solutions, when $\epsilon$ tends to zero, is a Pareto solution. Besides, many computer algorithms that exactly search for efficient points after finite numbers of steps only reach an approximate solution. Sometimes, obtaining exact solutions is impossible or very expensive in computational time. Thus, the search for approximate efficient points is relevant. In 1979, Kutateladze [15] introduced the concept of Pareto $\epsilon$-optimal solution. More recently, in 2010, Gutiérrez et al. [11] have studied the approximate solutions for the multiobjective optimization problem profusely.

Concerning the variational problems, firstly, in 1980, Giannessi [8] introduced the vector variational inequalities of Stampacchia type and secondly, in 1998, Giannessi [9] proposed Minty-type inequalities.

To achieve a solution to a vectorial optimization problem, we need appropriate convexity function concepts. In 2000, Ngai et al. [21] studied the concept of approximate convexity previously introduced in 1998 by Jofré et al. [13]. In this article, we will extend these generalized convex concepts to Hadamard manifolds. Besides, the design flight control architecture in aeroplanes uses generalized approximate geodesic functions and non-smooth optimization problem [7].

The environment in which we are going to study them is that of Hadamard manifolds. In non-linear spaces, such as the Hadamard manifolds, we extend concepts such as convex sets where geodesic arcs connect two points instead of linear segments. Mathematically, working with Hadamard manifolds has the advantage that sets that are not convex in the usual sense are convex within these manifolds. For example, the set

$$
X=\left\{\left(x_{1}, x_{2}\right) \in \mathbb{R}_{+}^{2}: x_{1}^{2}+x_{2}^{2} \leq 4 \leq\left(x_{1}-1\right)^{2}+x_{2}^{2}\right\}
$$

is not convex in the usual sense with $X \subset \mathbb{R}^{2}$, but $X$ is a geodesic convex on the Poincaré upper-plane model $\left(\mathbb{H}^{2}, g_{\mathbb{H}}\right)$, as it is the image of a geodesic segment. We can transform non-convex problems with Euclidean metrics into convex problems with related metrics [5]. In this way, we can take advantage of the excellent properties of convex sets. 
Within applications, a considerable number of optimization problems related to engineering [16], stereo vision processing [17], machine learning and computer vision in fields such as cancer tissue image analysis use Hadamard manifolds [6] [18]. The Hadamard manifolds are also used in the social sciences, for example, in Economics, within Game Theory, specifically in the achievement of Nash equilibrium points where strategy sets and payoff functions are geodesically convex, see [14].

In this article, we will look for Stackelberg's equilibrium points on Hadamard manifolds. Stackelberg games were introduced in 1934 (see [27]). The Stackelberg competition model is a game in which the leader player moves first, and then the follower player moves sequentially. Unlike Stackelberg's model, in the Nash model, the two players are competing with each other in the same level. In Novak et al. [22], the authors propose Stackelberg's model to describe the fight against terrorism.

State of the art is as follows.

Our paper has allowed us to extend another one from 2004, by Ruiz-Garzón et al. [23], where we established the relationships between variational-like inequality vector and optimization problems in Euclidean spaces.

The initial idea for this article comes from two groups of publications.

The first is articles dealing with the relations between vector variational inequality problems and non-smooth vector optimization problems using quasi efficient points in n-dimensional Euclidean spaces but not on Hadamard manifolds. Here would be the works of authors like Mishra and Upadhyay [19] in 2013 and Wang et al. [28] in 2017.

The other group of articles are the works in 2016 by Chen and Huang [3], Chen and Fang [2] in 2016 and Jayswal, Ahmad and Kumari [12] in 2019. This authors study the relations between vector optimization problems and vector variational inequalities and give some existence theorems for weakly efficient solutions on Hadamard manifolds but do not study the approximate solutions.

Hence, this paper comes to fill those two gaps by studying the relations between approximate solutions of vector optimization problems via solutions of variational-like inequalities problems on Riemannian manifolds.

More recently, in 2019, in Ruiz-Garzón et al. [25], we studied the constrained vector optimization problem as a particular case of the equilibrium vector with constraints problem on Hadamard manifolds.

In 2013, Nagy [20] studied the existence of Stackelberg equilibria point using appropriate variational inequalities in Euclidean spaces.

In 2019, Wang et al. [29] related the mixed variational inequality with the Nash equilibrium problem on Riemannian manifolds. This same year, RuizGarzón et al. [26] have demonstrated the coincidence of Nash's critical and equilibrium points on Hadamard context with generalized convex payoff functions. All this is what this article completes.

In 2020, Ansari, Islam and Yao [1] are making one of the latest efforts to relate variational and optimization problems where they have tested some existence results for non-smooth variational inequality problem and Minty non- 
smooth variational inequality problem using fixed point theorem on Hadamard manifolds. So, the topic of this article is hot.

Contributions. This work aims to fix the generalized geodesic convex conditions under which we can move from solutions of the variational-like inequalities problems to the local approximate weakly efficient solutions of the vector optimization problem or the Stackelberg equilibrium points because we have assured the coincidence of solutions and on surfaces that do not have to be linear like the Hadamard manifolds.

We have organized the contents of this paper as follows. Section 2 remember those elements typical of manifolds: geodesic curve, geodesic convex set, subdifferential or Hadamard manifold. In section 3, we will define the concepts of local approximate (weakly) efficient solution for Vector Optimization Problem on Hadamard manifolds. We will formulate the Stampacchia and Minty variational-like problems in their strong and weak form and introduce different concepts of generalized approximate geodesic convex functions and illustrate them with examples. In Section 4, we will relate approximate efficient points of Vector Optimization Problem to solutions of Stampacchia and Minty Variational-like Inequality Problems. We will characterize the approximate geodesic pseudoconvex and strictly pseudoconvex functions as the minimum requirement for all vector critical point vectors to be approximate weakly efficient and approximate efficient solutions, respectively. We will see that under generalized approximate geodesic convex conditions, we can identify critical points, solutions of vector variational-like problems and local approximate weakly efficient points of non-smooth vector optimization problems. In section 5, we will obtain the Stackelberg Equilibrium Problem via variational problems with geodesic convex loss functions. Finally, section 6 presents the conclusions to this study. Let us face our goal.

\section{Elements from manifolds}

We begin by presenting the environment of Hadamard manifolds in which we are going to move in this article. We recall some definitions and notions from manifolds. Let $M$ be a Riemannian manifold endowed with a Riemannian metric $g_{x}$ on a tangent space $T_{x} M$.

With the metric we can define:

(a) The corresponding norm is denoted by $\|\cdot\|_{x}$.

(b) The length of a piecewise $C^{1}$ curve $\gamma:[a, b] \rightarrow M$ is defined by

$$
L(\gamma)=\int_{a}^{b}\left\|\gamma^{\prime}(t)\right\|_{\gamma(t)} d t
$$

(c) The distance $d$ that induces the original topology on $M$, defined as

$$
d(x, y)=\inf \left\{L(\gamma) \mid \gamma \text { is a piecewise } C^{1} \text { curve joining } x \text { and } y, \forall x, y \in M\right\}
$$


This allows us to define the concept of minimal geodesic as any path $\gamma$ joining $x$ and $y$ in $M$ such that $L(\gamma)=d(x, y)$. If $M$ is complete, then any points in $M$ can be joined by a minimal geodesic.

This work is dedicated to the Hadamard manifolds, a particular case of Riemannian manifolds.

Definition 1 Recall that a simply connected complete Riemannian manifold of nonpositive sectional curvature is called a Hadamard manifold.

For differentiable manifolds, it is possible to define the derivatives of the curves on the manifold. The derivatives at a point $x$ on the manifold lie in a vector space $T_{x} M$. We denote by $T_{x} M$ the $n$-dimensional tangent space of $M$ at $x$, and denote by $T M=\bigcup_{x \in M} T_{x} M$ the tangent bundle of $M$.

Whereas $M$ is not a linear space, $T_{x} M$ is. Therefore, many proofs are based on transferring properties from the manifold to the tangent space and vice versa through two functions, the Riemannian exponential function and its inverse, $\exp$ and $\exp ^{-1}$, respectively.

Let $\bar{T} M$ be an open neighborhood of $M$ such that $\exp : \bar{T} M \rightarrow M$ is defined as $\exp _{x}(v)=\gamma_{v}(1, x)$ for every $v \in \bar{T} M$, where $\gamma_{v}$ is the geodesic starting at $x$ with velocity $v$ (i.e., $\gamma(0)=x, \gamma^{\prime}(0)=v$ ) [4]. It is easy to see that $\exp _{x}(t v)=\gamma_{v}(t, x)$.

The geodesic distance between $x$ and $y$ is $d(x, y)=\left\|\exp _{x}^{-1} y\right\|$. If $M=\mathbb{R}_{+}^{p}$ then $\exp _{x}^{-1} y=y-x$ and we denote by $\mathbb{R}_{+}^{p}$ the nonnegative orthant of $\mathbb{R}^{p}$, and $\mathbb{R}_{+}:=\mathbb{R}_{+}^{1}$.

When $M$ is a Hadamard manifold, then, $\exp _{x}$ is a diffeomorphism, and for any two points $x, y \in M$, there exists a unique minimal geodesic $\gamma_{x, y}(t)=$ $\exp _{x}\left(\operatorname{texp} p_{x}^{-1} y\right)$ for all $t \in[0,1]$ joining $x$ to $y$.

At this moment, we are in a position to define a generalization of the convex set concept to Hadamard environment:

Definition 2 A subset $X$ of $M$ is said to be a geodesic convex if, for any two points $x, y \in X$, the geodesic $\gamma$ of $M$ has endpoints $x$ and $y$ belonging to $X$; that is, if $\gamma:[0,1] \rightarrow M$ such that $\gamma(0)=x$ and $\gamma(1)=y$, then $\gamma_{x, y}(t)=\exp _{x}\left(\operatorname{texp}_{x}^{-1} y\right) \in X$.

We will also extend the concept of convex function:

Definition 3 Let $M$ be a Hadamard manifold and let $X \subseteq M$ be a geodesic convex set. A function $\theta: X \rightarrow \mathbb{R}$ is said to be convex if, for every $x, y \in X$,

$$
\theta\left(\gamma_{x, y}(t)\right) \leq t \theta(x)+(1-t) \theta(y), \quad \forall t \in[0,1]
$$

where $\gamma_{x, y}(t)=\exp _{y}\left(\operatorname{texp}_{y}^{-1} x\right)$ for every $t \in[0,1]$.

As we can see, we have replaced the segments with geodesic ones that join two points. Let us now recall the following concepts of Lipschitz function in the non-smooth case. 
Definition 4 A real-valued function $\theta$ defined on a Hadamard manifold $M$ is said to satisfy a Lipschitz condition of rank $k$ on a given subset $X$ of $M$ if $|\theta(x)-\theta(y)| \leq k d(x, y)$ for every $x, y \in X$.

A function $\theta$ is said to be Lipschitz near $x \in M$ if it satisfies the Lipschitz condition of some rank on an open neighborhood of $x$.

A function $\theta$ is said to be locally Lipschitz on $M$ if $\theta$ is Lipschitz near $x$ for every $x \in M$.

Example 1 The space of positive-definite matrices $S_{++}^{n}$ is an example of Hadamard manifold and with the Riemannian metric $\langle U, V\rangle=\left\langle X^{-1} U X^{-1}, V\right\rangle$ the function $\theta: S_{++}^{n} \rightarrow \mathbb{R}$ is Lipschitz on $S_{++}^{n}$.

In this article, we use non-smooth functions. With Lipschitz functions, generalized gradients or subdifferentials, replace the classical derivative.

Definition 5 [10] Let $\theta: M \rightarrow \mathbb{R}$ be a locally Lipschitz function on a Hadamard manifold $M$. We define the subdifferential of $\theta$ at $x$, denoted by $\partial \theta(x)$, as the subset of $T_{x} M$ with the support function given by $\theta^{0}(x ;$.$) , i.e.,$ for every $v \in T_{x} M$,

$$
\theta^{0}(x, v)=\sup \{\langle A, v\rangle: A \in \partial \theta(x)\}
$$

It can be proved that the generalized Jacobian is

$$
\partial \theta(x)=\operatorname{conv}\left\{\lim _{i \rightarrow \infty} \operatorname{grad} \theta\left(x_{i}\right):\left\{x_{i}\right\} \subseteq X, x_{i} \rightarrow x\right\}
$$

where $X$ is a dense subset of $M$ on which $\theta$ is differentiable.

We briefly examine some particular cases:

Obviously, if $\theta$ is differentiable at $x \in M$, we define the gradient of $\theta$ as the unique vector $\operatorname{grad} \theta(x) \in T_{x} M$ that satisfies

$$
d \theta_{x}(v)=\langle\operatorname{grad} \theta(x), v\rangle, \quad \forall v \in T_{x} M
$$

And when $\theta$ is a locally Lipschitz convex function, we have $\theta^{0}(x ; v)=$ $\theta^{\prime}(x ; v)$ for all $x \in M$. For a convex function $\theta: M \rightarrow \mathbb{R}$, the directional derivative of $\theta$ at the point $x \in M$ in the direction $v \in T_{y} M$ is defined by

$$
\theta^{\prime}(x, v)=\lim _{t \rightarrow 0^{+}} \frac{\theta\left(\exp _{x}(t v)\right)-\theta(x)}{t}
$$

and the subdifferential of $\theta$ at $x$ is

$$
\partial \theta(x)=\left\{A \in T_{x} M \mid \theta^{\prime}(x ; v) \geq\langle A, v\rangle, \forall v \in T_{x} M\right\}
$$

However, for the vector function $f=\left(f_{1}, \ldots, f_{p}\right): M \rightarrow \mathbb{R}^{p}$, the generalized Jacobian $\partial f(x)$ is contained and, in general, is different from the Cartesian product of Clarke subdifferentials of the components of $f$.

So far all the math tools to work on Hadamard manifolds. 


\section{Definitions and formulations}

In this section, we consider the unconstrained multiobjective programming problem (VOP) defined as:

$$
\begin{array}{ll}
\text { (VOP) } & \min f(x) \\
& \text { s.t. } x \in X \subseteq M
\end{array}
$$

where $f=\left(f_{1}, \ldots f_{p}\right): X \subseteq M \rightarrow \mathbb{R}^{p}$, with $f_{i}: X \subseteq M \rightarrow \mathbb{R}$ for all $i: 1, \ldots, p$, locally Lipschitz functions on the open set $X \subseteq M$, and $M$ assumed to be a Hadamard manifold.

In this formulation, the value of the variable is not necessarily a point in Euclidean space but for example, a positive-definite matrix. For the Vectorial Optimization Problem, we will define concepts close to efficiency.

The following concepts are an extension to Hadamard manifolds of others defined by Mishra and Upadhyay [19] and Wang et al. [28] in linear spaces.

Definition 6 A feasible point $\bar{x}$ is said to be:

(a) A local approximate efficient (AE) solution for VOP if there exists $\alpha \in$ $\operatorname{int}\left(\mathbb{R}_{+}^{p}\right)$ and a neighborhood $B(\bar{x}, \delta)$ of $\bar{x}$ such that does not exist another feasible point $x \in B(\bar{x}, \delta) \cap X$ such that

$$
f(x)-f(\bar{x})+\alpha\left\|\exp _{\bar{x}}^{-1} x\right\| \in-\mathbb{R}_{+}^{p} \backslash\{0\}
$$

(b) A local approximate weakly efficient (AWE) solution for VOP if there exists $\alpha \in \operatorname{int}\left(\mathbb{R}_{+}^{p}\right)$ and a neighborhood $B(\bar{x}, \delta)$ of $\bar{x}$ such that does not exist another feasible point $x \in B(\bar{x}, \delta) \cap X$ such that

$$
f(x)-f(\bar{x})+\alpha\left\|\exp _{\bar{x}}^{-1} x\right\| \in-\operatorname{int} \mathbb{R}_{+}^{p}
$$

Obviously, all efficient (resp. weakly efficient) point is approximate efficient (resp. weakly efficient). The reverse may not be true.

Example 2 Let $M=\mathbb{R}_{++}=\{x \in \mathbb{R}: x>0\}$ be endowed with the Riemannian metric defined by $g(x)=x^{-2}$. It is well known that $M$ is a Hadamard manifold.

The geodesic curve $\gamma: \mathbb{R} \rightarrow M$ with the initial condition $\gamma(0)=x$ and $\gamma^{\prime}(0)=w \in T_{x} M$ is given by $\gamma(t)=x e^{(w / x) t}, \operatorname{implies}$ that $\exp _{x}(t w)=x e^{(w / x) t}$ and it follows that $\exp _{x}^{-1} y=x \ln \left(\frac{y}{x}\right)$. The Riemannian distance is given by $d(x, y)=\left\|\exp _{x}^{-1} y\right\|=\left|\ln \left(\frac{x}{y}\right)\right|$.

Consider the VOP as follows:

$$
\begin{gathered}
\min f(x)=\left(f_{1}(x), f_{2}(x)\right)=(\ln (x+1), \ln (x+2)) \\
\text { s.t. } \quad x \in X=\left\{x \mid x=e^{t}, t \in[0,1]\right\} \subset M
\end{gathered}
$$

For $\bar{x}=1.5$, there exists another feasible point $x=1.4 \in B(\bar{x}, \delta) \cap X$ such that

$$
f(x)-f(\bar{x})=(\ln (2.4), \ln (3.4))-(\ln (2.5), \ln (3.5))=(-0.04,-0.03)<(0,0)
$$

Hence, $\bar{x}=1.5$ is not a weak efficient solution for VOP. 
Now, it is easy to see that for any $\alpha=(1,1)>0$, there exists $\delta>0$ such that, for all $x \in B(x, \delta)$, does not exist another feasible point $x \in B(\bar{x}, \delta) \cap X$ such that

$$
f(x)-f(\bar{x})+\alpha\left\|\exp _{\bar{x}}^{-1} x\right\| \in-\operatorname{int} \mathbb{R}_{+}^{p}
$$

Therefore, $\bar{x}=1.5$ is a local approximate weakly efficient (AWE) solution for VOP.

Variational problem solving is an intermediate step in solving optimization problems since their solutions coincide under convexity assumptions. We can study the vector optimization problem via the vector variational-like inequality problems.

We define the Stampacchia and Minty version of these vector variationallike problems on Hadamard manifold. We consider these problems as "primal" and "dual" version problems due to solutions' relations, in the same way, that it happens for the mathematical programming problem. Generally, the Minty type formulation is more accessible to resolve than the Stampacchia type.

\section{Definition $7[3]$}

(a) Stampacchia Vector Variational-Like Inequality Problem (SV): Find a point $\bar{x} \in X$ such that there exists no $x \in X \subset M$ such that

$$
\left\langle A, \exp _{\bar{x}}^{-1} x\right\rangle \leq 0, \quad \forall A \in \partial f(\bar{x})
$$

(b) Stampacchia Weak Vector Variational-Like Inequality Problem (SWV): Find a point $\bar{x} \in X$ such that there exists no $x \in X$ such that

$$
\left\langle A, \exp _{\bar{x}}^{-1} x\right\rangle<0, \quad \forall A \in \partial f(\bar{x})
$$

(c) Minty Vector Variational-Like Inequality Problem (MV): Find a point $\bar{x} \in$ $X$ such that there exists no $x \in X$ such that

$$
\left\langle C, \exp _{x}^{-1} \bar{x}\right\rangle \geq 0, \quad \forall C \in \partial f(x)
$$

(d) Minty Weak Vector Variational-Like Inequality Problem (MWV): Find a point $\bar{x} \in X$ such that there exists no $x \in X$ such that

$$
\left\langle C, \exp _{x}^{-1} \bar{x}\right\rangle>0, \quad \forall C \in \partial f(x)
$$

Under conditions of generalized convexity it is possible to move from the solution of a vector variational-like problem to another, for this we need the following definitions of generalized convexity on Hadamard manifolds.

Inspired by the work of Ngai, Luc and Thera [21] where they introduced the concept of approximate convex functions, we present generalized approximate geodesic convex functions on Hadamard manifolds:

Definition 8 Let $M$ be a Hadamard manifold, $X$ is an open geodesic convex subset of $M$ and $f: X \subseteq M \rightarrow \mathbb{R}^{p}$ is a locally Lipschitz function. The function $f$ is said to be: 
(a) approximate geodesic convex (AGCX) at $\bar{x} \in X$ if for all $\alpha \in \operatorname{int}\left(\mathbb{R}_{+}^{p}\right)$ there exists $\delta>0$ such that $\forall x \in B(\bar{x}, \delta) \cap X, \forall A \in \partial f(\bar{x})$ we have

$$
f(x)-f(\bar{x})-\left\langle A, \exp _{\bar{x}}^{-1} x\right\rangle+\alpha\left\|\exp _{\bar{x}}^{-1} x\right\| \in \mathbb{R}_{+}^{p}
$$

(b) approximate geodesic strictly convex (AGSCX) at $\bar{x}$ if for all $\alpha \in \operatorname{int}\left(\mathbb{R}_{+}^{p}\right)$ there exists $\delta>0$ such that $\forall x \in B(\bar{x}, \delta) \cap X, \forall A \in \partial f(\bar{x})$ we have

$$
f(x)-f(\bar{x})-\left\langle A, \exp _{\bar{x}}^{-1} x\right\rangle+\alpha\left\|\exp _{\bar{x}}^{-1} x\right\| \in \operatorname{int} \mathbb{R}_{+}^{p} .
$$

(c) approximate geodesic pseudoconvex (AGPCX) at $\bar{x}$ if for all $\alpha \in \operatorname{int}\left(\mathbb{R}_{+}^{p}\right)$ there exists $\delta>0$ such that $\forall x \in B(\bar{x}, \delta) \cap X, \forall A \in \partial f(\bar{x})$ we have

$$
f(x)-f(\bar{x})+\alpha\left\|\exp _{\bar{x}}^{-1} x\right\| \in-\operatorname{int} \mathbb{R}_{+}^{p} \Rightarrow\left\langle A, \exp _{\bar{x}}^{-1} x\right\rangle \in-\operatorname{int} \mathbb{R}_{+}^{p} .
$$

(d) approximate geodesic strictly pseudoconvex (AGSPCX) at $\bar{x}$ if for all $\alpha \in$ $\operatorname{int}\left(\mathbb{R}_{+}^{p}\right)$ there exists $\delta>0$ such that $\forall x \in B(\bar{x}, \delta) \cap X, \forall A \in \partial f(\bar{x})$ we have

$$
f(x)-f(\bar{x})+\alpha\left\|\exp _{\bar{x}}^{-1} x\right\| \in-\mathbb{R}_{+}^{p} \backslash\{0\} \Rightarrow\left\langle A, \exp _{\bar{x}}^{-1} x\right\rangle \in-\operatorname{int} \mathbb{R}_{+}^{p} .
$$

The function $f$ is said to be approximate geodesic convex (resp. strictly convex, pseudoconvex, strictly pseudoconvex) on $X$ if, for every $x \in X, f$ is approximate geodesic convex (resp. strictly convex, pseudoconvex, strictly pseudoconvex) at $x$ on $X$.

The following examples illustrate the above definitions and relations on Hadamard manifolds.

Example 3 Let $M=\mathbb{R}_{++}=\{x \in \mathbb{R}: x>0\}$ be endowed with the Riemannian metric defined by $g(x)=x^{-2}$, where $X=\left\{x \mid x=e^{t}, t \in[0,1]\right\} \subset M$.

Let $f(x)=\left(f_{1}, f_{2}\right)(x): X \subset M=\mathbb{R}_{++} \rightarrow \mathbb{R}^{2}$ be a function with

$$
f_{1}(x)=x \quad \text { and } \quad f_{2}(x)=\left\{\begin{array}{lll}
-x+\frac{3}{2} & \text { if } & x<1.5 \\
0 & \text { if } & 1.5 \leq x \leq 2 \\
x-2 & \text { if } & x>2
\end{array}\right.
$$

The function $f$ is approximate geodesic convex on $M$ because its components are linear functions.

Example 4 Let $f(x)=\left(f_{1}, f_{2}\right)(x): X \subset M=\mathbb{R}_{++} \rightarrow \mathbb{R}^{2}$ be a function defined as

$$
f_{1}(x)=x \quad \text { and } \quad f_{2}(x)=\left\{\begin{array}{lll}
-x+1.5, & \text { if } & x<1.5 \\
0, & \text { if } & 1.5 \leq x \leq 2 \\
2-x, & \text { if } & x>2
\end{array}\right.
$$

where $X=\left\{x \mid x=e^{t}, t \in[0,1]\right\} \subset M$ and we can calculate

$$
\partial f(x)= \begin{cases}\left(x^{2},-x^{2}\right) & \text { if } x<1.5 \text { or } x>2 \\ \left(x^{2}, a x^{2}\right) & \text { if } x=1.5 \text { or } x=2 \text { with }-1 \leq a \leq 0 \\ \left(x^{2}, 0\right) & \text { if } 1.5<x<2 .\end{cases}
$$

The function $f$ is approximate geodesic pseudoconvex on $X$ because $f(x)-$ $f(\bar{x})+\left\|\exp _{\bar{x}}^{-1} x\right\| \in-\operatorname{int} \mathbb{R}_{+}^{2}$ implies that $f$ should be nondecreasing, but $f_{2}$ is nonincreasing and this previous condition is not satisfied. 
Example 5 Let $f(x)=\left(f_{1}, f_{2}\right)(x): X \subset M=\mathbb{R}_{++} \rightarrow \mathbb{R}^{2}$ be a function with $f(x)=(|x-1|,-|x-1|)$, where $X=\left\{x \mid x=e^{t}, t \in[0,1]\right\} \subset M$. The function $f$ is approximate geodesic strictly pseudoconvex because $f(x)-f(\bar{x})+$ $\alpha\left\|\exp _{\bar{x}}^{-1} x\right\| \in-\mathbb{R}_{+}^{p} \backslash\{0\}$ is not satisfied for $x \in X \subset M$.

We now have all the tools required to discuss approximate solutions of vector optimization problems and vector variational-like inequalities problems.

\section{Approximate solutions of vector optimization problems via vector variational-like inequality problems}

This section aims to see how to relate the solutions of these problems on Hadamard manifolds.

We will set out by endeavouring to calculate the approximate efficient points, starting with the solutions to the Stampacchia Vector Variational-Like Inequality Problem.

Theorem 1 Let $M$ be a Hadamard manifold, $X$ is an open geodesic convex subset of $M$, and $f: X \subseteq M \rightarrow \mathbb{R}^{p}$ is a locally Lipschitz and approximate geodesic convex (AGCX) function at $\bar{x} \in X$. If $\bar{x}$ solves the Stampacchia Vector Variational-Like Inequality Problem $(S V)$, then $\bar{x}$ is a local approximate efficient (AE) point to the Vector Optimization Problem (VOP).

Proof. Suppose that $\bar{x}$ is not a local approximate efficient (AE) point to $V O P$ then there exists $\alpha \in \operatorname{int}\left(\mathbb{R}_{+}^{p}\right)$ and a neighborhood $B(\bar{x}, \delta)$ of $\bar{x}$ such that there exists another feasible point $x \in B(\bar{x}, \delta) \cap X$ such that

$$
f(x)-f(\bar{x})+\alpha\left\|\exp _{\bar{x}}^{-1} x\right\| \in-\mathbb{R}_{+}^{p} \backslash\{0\}
$$

Since $f$ is AGCX function at $\bar{x}$, we have ensured that if for all $\alpha \in \operatorname{int}\left(\mathbb{R}_{+}^{p}\right)$ there exists $\delta>0$ such that $\forall x \in B(\bar{x}, \delta) \cap X, \forall A \in \partial f(\bar{x})$ we have

$$
\left\langle A, \exp _{\bar{x}}^{-1} x\right\rangle \leq 0
$$

therefore $\bar{x}$ is not a solution to $S V$. Contradiction.

The above result extends the Theorem 3.2 given by Wang et al. [28] and Mishra and Upadhyay [19] in Euclidean spaces to Hadamard manifolds and the Theorem 3.4 given in Jayswal et al. [12] to approximate solutions.

Thus is, under conditions of approximate geodesic convexity functions, the solutions of the Stampacchia Vector Variational-Like Inequality Problem are local approximate efficient points. To prove the sufficient condition, we need to impose stronger assumptions on the approximate geodesic convexity of functions: 
Theorem 2 Let $M$ be a Hadamard manifold, $X$ is an open geodesic convex subset of $M$, and $f: X \subseteq M \rightarrow \mathbb{R}^{p}$ is a locally Lipschitz and that $-f$ is approximate geodesic strictly convex $(A G S C X)$ function. If $\bar{x}$ is a local approximate weakly efficient point $(A W E)$ then $\bar{x}$ also solves the Stampacchia Vector Variational-Like Inequality Problem $(S V)$.

Proof. Suppose that $\bar{x}$ is a local AWE solution for $V O P$, but not the $S V$. Then, $\exists x \in X$ and $A \in \partial f(\bar{x})$ such that $\left\langle A, \exp _{\bar{x}}^{-1} x\right\rangle \leq 0$.

By the approximate geodesic strictly convexity $(A G S C X)$ of $-f$, we have that for all $\alpha \in \operatorname{int}\left(\mathbb{R}_{+}^{p}\right)$ there exists $\delta>0$ such that $\forall x \in B(\bar{x}, \delta) \cap X, \forall A \in$ $\partial f(\bar{x})$ we have

$$
f(x)-f(\bar{x})+\alpha\left\|\exp _{\bar{x}}^{-1} x\right\|<\left\langle A, \exp _{\bar{x}}^{-1} x\right\rangle \leq 0
$$

$\bar{x}$ being a local AWE point, what is a contradiction.

Corollary 1 Let $M$ be a Hadamard manifold, $X$ is an open geodesic convex subset of $M$, and $f: X \subseteq M \rightarrow \mathbb{R}^{p}$ is a locally Lipschitz and $-f$ is approximate geodesic strictly convex $(A G S C X)$ function. If $\bar{x}$ is a local approximate efficient $(A E)$ point then $\bar{x}$ solves the Stampacchia Vector Variational-Like Inequality Problem $(S V)$.

Proof. Because every local AE point is a local AWE point and by the previous theorem 2 , it would be proved.

Let us now see what the relationship is between the local approximate efficient solutions of $V O P$ and the solutions of Minty Vector Variational-Like Inequality Problem $(M V)$ :

Theorem 3 Let $M$ be a Hadamard manifold, $X$ is an open geodesic convex subset of $M$, and $f: X \subseteq M \rightarrow \mathbb{R}^{p}$ is a locally Lipschitz and $f$ is approximate geodesic convex $(A G C X)$ function on $X$. If $\bar{x}$ is a local approximate efficient $(A E)$ for the Vector Optimization Problem $(V O P)$ then $\bar{x}$ solves the Minty Vector Variational-Like Inequality Problem $(M V)$.

Proof. By contradiction ad absurdum. Suppose that $\bar{x}$ does not solve MV then there exists $x \in X$ satisfying

$$
\left\langle C, \exp _{x}^{-1} \bar{x}\right\rangle \geq 0, \quad \forall C \in \partial f(x)
$$

Noticing that $f$ is AGCX, for all $\alpha \in \operatorname{int}\left(\mathbb{R}_{+}^{p}\right)$ there exists $\delta>0$ such that $\forall \bar{x} \in B(x, \delta) \cap X, \forall C \in \partial f(x)$ such that

$$
f(\bar{x})-f(x)-\left\langle C, \exp _{x}^{-1} \bar{x}\right\rangle+\alpha\left\|\exp _{x}^{-1} \bar{x}\right\| \in \mathbb{R}_{+}^{p} .
$$

If follows that

$$
f(\bar{x})-f(x)+\alpha\left\|\exp _{x}^{-1} \bar{x}\right\| \geq 0
$$

which leads to a contradiction, since $\bar{x}$ is an AE solution. 
Then, in an environment of generalized approximate geodesic convexity we have to:

$$
\mathrm{SV} \Leftrightarrow \mathrm{AE} \Rightarrow \mathrm{MV}
$$

Thus is, the generalized approximate geodesic convexity allows us to relate solutions of SV and MV problems and local approximate efficient solutions of the vector optimization problem on Hadamard manifolds as an extension of what happened in Euclidean spaces, see Ruiz-Garzón et al. [23].

We will go one step further, and we will look for when we can identify solutions to Stampacchia Weak Vector Variational-Like Inequality Problem $(S W V)$ with the local approximate weakly efficient $(A W E)$ points for VOP.

Theorem 4 Let $M$ be a Hadamard manifold, $X$ is an open geodesic convex subset of $M$, and $f: X \subseteq M \rightarrow \mathbb{R}^{p}$ is a locally Lipschitz function.

If $\bar{x}$ is local approximate weakly efficient $(A W E)$ for the Vector Optimization Problem $(V O P)$ then $\bar{x}$ solves the Stampacchia Weak Vector VariationalLike Inequality Problem $(S W V)$.

If $f$ is a approximate geodesic pseudoconvex (AGPCX) function and $\bar{x}$ solves the $S W V$ problem then $\bar{x}$ is a local AWE point for VOP.

Proof.

Firstly, we will prove the necessary condition. Let $\bar{x}$ be the local AWE solution of $V O P$, since $X$ is an geodesic convex set, there exists $\alpha \in \operatorname{int}\left(\mathbb{R}_{+}^{p}\right)$ and a neighborhood $B(\bar{x}, \delta)$ of $\bar{x}$ such that there does not exist another feasible point $x \in B(\bar{x}, \delta) \cap X$ such that

$$
f\left(\exp _{\bar{x}}\left(\operatorname{texp}_{\bar{x}}^{-1} x\right)\right)-f(\bar{x})<-\alpha t\left\|\exp _{\bar{x}}^{-1} x\right\| \in-\mathbb{R}_{+}^{p}, \quad 0<t<1
$$

Dividing the above inequality by $t$ and taking the limit as $t$ tends to 0 , we get to does not exist $x \in M$ such that $\left\langle A, \exp _{\bar{x}}^{-1} x\right\rangle<0, \forall A \in \partial f(\bar{x})$ then $\bar{x}$ solve $S W V$.

Secondly, we will prove the reciprocal condition by reductio ad absurdum. If $\bar{x}$ is not a local AWE point then there exists $\alpha \in \operatorname{int}\left(\mathbb{R}_{+}^{p}\right)$ and a neighborhood $B(\bar{x}, \delta)$ of $\bar{x}$ such that there exists another feasible point $x \in B(\bar{x}, \delta) \cap X$ such that

$$
f(x)-f(\bar{x})+\alpha\left\|\exp _{\bar{x}}^{-1} x\right\| \in-\operatorname{int} \mathbb{R}_{+}^{p}
$$

By AGPCX of $f$ we have ensured that for all $\alpha \in \operatorname{int}\left(\mathbb{R}_{+}^{p}\right)$ there exists $\delta>0$ such that $\forall x \in B(\bar{x}, \delta) \cap X, \forall A \in \partial f(\bar{x})$ we have

$$
\left\langle A, \exp _{\bar{x}}^{-1} x\right\rangle \in-\operatorname{int} \mathbb{R}_{+}^{p} .
$$

Contradiction, with $\bar{x}$ is a solution of the SWV.

Remark that Wang et al. [28] only proves the second part of the above theorem and not for Hadamard manifolds. Theorem 4.3 given in Ruiz-Garzón et al. [23] in n-dimensional Euclidean space is a particular case of the result proven here.

Every approximate efficient point is approximate weakly efficient, but when the reverse condition occurs. The following theorem proves it: 
Theorem 5 Let $M$ be a Hadamard manifold, $X$ is an open geodesic convex subset of $M, f: X \subseteq M \rightarrow \mathbb{R}^{p}$ is a locally Lipschitz and approximate geodesic strictly convex $(A G S C X)$ function on $X$. If $\bar{x}$ is a local approximate weakly efficient $(A W E)$ for Vector Optimization Problem then $\bar{x}$ is a local approximate efficient (AE) for Vector Optimization Problem (VOP).

Proof. Suppose that $\bar{x}$ is a local AWE point for VOP, but not a local AE point. Then, there exists $\alpha \in \operatorname{int}\left(\mathbb{R}_{+}^{p}\right)$ and a neighborhood $B(\bar{x}, \delta)$ of $\bar{x}$ such that there exists another feasible point $x \in B(\bar{x}, \delta) \cap X$ such that

$$
f(x)-f(\bar{x})+\alpha\left\|\exp _{\bar{x}}^{-1} x\right\| \in-\mathbb{R}_{+}^{p} \backslash\{0\}
$$

By the AGSCX of $f$ we have that for all $\alpha \in \operatorname{int}\left(\mathbb{R}_{+}^{p}\right)$ there exists $\delta>0$ such that $\forall x \in B(\bar{x}, \delta) \cap X, \forall A \in \partial f(\bar{x})$ we have

$$
f(x)-f(\bar{x})-\left\langle A, \exp _{\bar{x}}^{-1} x\right\rangle+\alpha\left\|\exp _{\bar{x}}^{-1} x\right\|>0
$$

which is to say, $\exists x \in X$, such that $\left\langle A, \exp _{\bar{x}}^{-1} x\right\rangle<0, \quad \forall A \in \partial f(\bar{x})$, therefore, $\bar{x}$ does not solve the SWV problem.

The contradiction arises from, on the other hand, the earlier theorem 4, we have that if $\bar{x}$ is a local AWE for VOP then $\bar{x}$ solves also the SWV.

We know that we look for among the critical points to find solutions to optimization problems. In Ruiz-Garzón et al. [24] the following definition is given:

Definition 9 Let $M$ be a Hadamard manifold, $X$ is an open geodesic convex subset of $M$, and $f: X \subseteq M \rightarrow \mathbb{R}^{p}$ is a locally Lipschitz function. A feasible point $\bar{x} \in X$ is said to be a vector critical point (VCP) if there exist some $x \in X \subseteq M$ and $\lambda \in \mathbb{R}_{+}^{p} \backslash\{0\}$ such that

$$
\lambda^{T}\left\langle A, \exp _{\bar{x}}^{-1} x\right\rangle=0 \text { for some } A \in \partial f(\bar{x})
$$

In the following theorem, we show that the key to the relationship between vectorial critical points $(V C P)$ and local AWE solutions for VOP is the approximate geodesic pseudoconvexity $(A G P C X)$ :

Theorem 6 Let $M$ be a Hadamard manifold, $X$ is an open geodesic convex subset of $M$, and $f: X \subseteq M \rightarrow \mathbb{R}^{p}$ is a locally Lipschitz function. Every $(V C P)$ is a local approximate weakly efficient solution $(A W E)$ for $V O P$ if and only if the function $f$ is approximate geodesic pseudoconvex (AGPCX) on $X$.

Proof Firstly, we prove that if very VCP is a local approximate weakly efficient solution AWE for VOP then the function $f$ is AGPCX at $\bar{x}$.

Let $\bar{x}$ be a local AWE for VOP then there exists $\alpha \in \operatorname{int}\left(\mathbb{R}_{+}^{p}\right)$ and a neighborhood $B(\bar{x}, \delta)$ of $\bar{x}$ such that

$$
f(x)-f(\bar{x})<-\alpha\left\|\exp _{\bar{x}}^{-1} x\right\|
$$


has no solution $x \in B(\bar{x}, \delta) \cap X$.

On the other hand, if $\bar{x}$ is a vector critical point for some $x \in X \subseteq M$, $\lambda \in \mathbb{R}_{+}^{p} \backslash\{0\}$, and $A \in \partial f(\bar{x})$ we have

$$
\lambda^{T}\left\langle A, \exp _{\bar{x}}^{-1} x\right\rangle=0
$$

By Gordan's Alternative Theorem there exists a vector $A \in \partial f(\bar{x})$ such that the following system

$$
\lambda^{T}\left\langle A, \exp _{\bar{x}}^{-1} x\right\rangle<0
$$

has no solution.

Therefore, the systems (1) and (2) are equivalent, hence, for all $\alpha \in \operatorname{int}\left(\mathbb{R}_{+}^{p}\right)$ there exists $\delta>0$ such that $\forall x \in B(\bar{x}, \delta) \cap X, \forall A \in \partial f(\bar{x})$ we have

$$
f(x)-f(\bar{x})+\alpha\left\|\exp _{\bar{x}}^{-1} x\right\| \in-\operatorname{int} \mathbb{R}_{+}^{p} \Rightarrow\left\langle A, \exp _{\bar{x}}^{-1} x\right\rangle \in-\operatorname{int} \mathbb{R}_{+}^{p} .
$$

Thus is, $f$ is AGPCX at $\bar{x}$.

We prove the sufficient condition. We assume by hypothesis that $f$ is AGPCX and that $\bar{x}$ is a VCP. Thus,

$$
\lambda^{T}\left\langle A, \exp _{\bar{x}}^{-1} x\right\rangle=0
$$

for some $x \in X \subseteq M, \lambda \in \mathbb{R}_{+}^{p} \backslash\{0\}$, and $A \in \partial f(\bar{x})$ and we need to prove that $\bar{x}$ is a local AWE point. By reductio ad absurdum, suppose that $\bar{x}$ is not a local weakly approximate efficient solution AWE for VOP. Then, there exists another feasible point $x \in B(\bar{x}, \delta) \cap X$ such that

$$
f(x)-f(\bar{x})+\alpha\left\|\exp _{\bar{x}}^{-1} x\right\| \in-\operatorname{int} \mathbb{R}_{+}^{p}
$$

Using the fact that $f$ is AGPCX at $\bar{x}$ on $X$, we have $\left\langle A, \exp _{\bar{x}}^{-1} x\right\rangle \in$ $-\operatorname{int} \mathbb{R}_{+}^{p}$, and so

$$
\lambda^{T}\left\langle A, \exp _{\bar{x}}^{-1} x\right\rangle<0
$$

which contradicts (3).

Therefore, we have extended the theorem 4.2 given by Wang et al. [28], the theorem 3.5 given by Mishra and Upadhyay [19] and the theorem 4.5 given by Ruiz-Garzón et al. [23] for Euclidean spaces to Hadamard manifolds with local approximate weakly efficient (AWE) points and approximate geodesic pseudoconvex (AGPCX) functions.

For local approximate efficient solutions, the approximate geodesic strictly approximate pseudoconvexity $(A G S P C X)$ replaces the role of approximate geodesic pseudoconvexity $(A G P C X)$. Thus, in the same way, we can prove the following corollary.

Corollary 2 Let $M$ be a Hadamard manifold, $X$ is an open geodesic convex subset of $M$, and $f: X \subseteq M \rightarrow \mathbb{R}^{p}$ is a locally Lipschitz function. Every $V C P$ is a local approximate efficient solution (AE) for VOP if and only if the function $f$ is approximate geodesic strictly pseudoconvex (AGSPCX) on $X$. 
We must emphasize that Theorem 6 and Corollary 2 show that approximate geodesic pseudoconvexity (resp. approximate geodesic strictly pseudoconvexity) is a minimal requirement for the property that every VCP is a local weakly approximate efficient (resp. approximate efficient) solution of problem VOP on a Hadamard manifold in the non-smooth case.

In summary, we have that

$$
\begin{gathered}
[\mathrm{VCP} \Leftrightarrow \mathrm{AWE}] \Leftrightarrow \mathrm{AGPCX}] \\
[\mathrm{VCP} \Leftrightarrow \mathrm{AE}] \Leftrightarrow \mathrm{AGSPCX}]
\end{gathered}
$$

The following theorem tries to check what the relationships are between Stampacchia and Minty weak type problems.

Theorem 7 Let $M$ be a Hadamard manifold, $X$ is an open geodesic convex subset of $M, f: X \subseteq M \rightarrow \mathbb{R}^{p}$ is a locally Lipschitz and approximate geodesic convex $(A G C X)$ function. If $\bar{x}$ is a local approximate weakly efficient $(A W E)$ point then $\bar{x}$ solves Minty Weak Vector Variational-Like Inequality Problem $(M W V)$.

Proof. By reductio ad absurdum. Suppose that $\bar{x}$ is not a solution for (MWV), then there exists $x \in X$ such that

$$
\left\langle C, \exp _{x}^{-1} \bar{x}\right\rangle>0, \quad \forall C \in \partial f(x)
$$

Since $f$ is AGCX on $\mathrm{X}$, for all $\alpha \in \operatorname{int}\left(\mathbb{R}_{+}^{p}\right)$ there exists $\delta>0$ such that $\forall x \in B(\bar{x}, \delta) \cap X, \forall C \in \partial f(x)$ we have

$$
f(\bar{x})-f(x)-\left\langle C, \exp _{x}^{-1} \bar{x}\right\rangle+\alpha\left\|\exp _{x}^{-1} \bar{x}\right\| \in \mathbb{R}_{+}^{p} .
$$

From (4) and (5), there exists another feasible point $x \in B(\bar{x}, \delta) \cap X$ such that

$$
f(\bar{x})-f(x)+\alpha\left\|\exp _{x}^{-1} \bar{x}\right\|>0
$$

which gives us a contradiction that $\bar{x}$ is a AWE point for VOP.

We also know that:

Theorem 8 Let $M$ be a Hadamard manifold, $X$ is an open geodesic convex subset of $M, f: X \subseteq M \rightarrow \mathbb{R}^{p}$ is a locally Lipschitz. The point if $\bar{x}$ solves Minty Weak Vector Variational-Like Inequality Problem $(M W V)$ then $\bar{x}$ solves the Stampacchia Weak Vector Variational-Like Inequality Problem $(S W V)$.

Proof. This condition is the theorem 3.4 proved in Chen and Huang [3].

Therefore, the relationship between SWV and MWV problems on Hadamard manifolds is maintained. This relationship is at the base of the theorems of existence for local approximate weak efficient solutions for VOP, for which it is sufficient to prove that SWV problem has a solution.

Moreover, we obtain as a final result: 
Corollary 3 Let $M$ be a Hadamard manifold, $X$ is an open geodesic convex subset of $M, f: X \subseteq M \rightarrow \mathbb{R}^{p}$ is a locally Lipschitz and approximate geodesic convex $(A G C X)$. The point $\bar{x}$ is a local approximate weakly efficient (AWE) if and only if is a Vector Critical Point (VCP) if and only if $\bar{x}$ solves a Stampacchia Weak Vector Variational-Like Inequality Problem (WSV) if and only if $\bar{x}$ solves a Minty Weak Vector Variational-Like Inequality Problem (WMV).

Proof. It is the result of applying the theorems 4, 6, 7 and 8 and that AGCX implies AGPCX.

To sum up:

$$
\mathrm{VCP} \Leftrightarrow \mathrm{SWV} \Leftrightarrow \mathrm{AWE} \Leftrightarrow \mathrm{MWV}
$$

The above theorem is an extension to local approximative weak efficient (AWE) points of the relationships obtained by Chen and Huang [3] in Theorem 3.8 for weakly efficient points.

Under approximate geodesic pseudoconvex conditions, we can identify Vector Critical Points, approximate weakly efficient points of VOP and solutions of Weak Stamppacchia Variational-like inequalities problems just as we did with finite-dimensional Euclidean spaces. Let us look at an illustrative example.

Example 6 Let $M=\mathbb{R}_{++}=\{x \in \mathbb{R}: x>0\}$ be endowed with the Riemannian metric defined by $g(x)=x^{-2}$. It is well known that $\exp _{x}^{-1} y=x \ln \left(\frac{y}{x}\right)$ and the Riemannian distance is given by $d(x, y)=\left\|\exp _{x}^{-1} y\right\|=\left|\ln \left(\frac{x}{y}\right)\right|$.

Consider:

$(\mathrm{VOP}) \quad \min f(x)=\left(f_{1}, f_{2}\right)(x)$

$$
\text { subject to } \quad x \in X=\left\{x \mid x=e^{t}, t \in[0,1]\right\} \subset M
$$

where let $f$ be a function defined as

$$
f_{1}(x)=\ln (x) \text { and } f_{2}(x)=\left\{\begin{array}{lll}
0, & \text { if } & x<2 \\
x-2, & \text { if } & x>2
\end{array}\right.
$$

and we can calculate

$$
\partial f_{1}(x)=\operatorname{grad} f_{1}(x)=g(x)^{-1} \frac{\partial f_{1}}{\partial x}=x^{2} \frac{1}{x}=x
$$

similarly, we get

$$
\partial f_{2}(x)=\left\{\begin{array}{lll}
0 & \text { if } & x<2 \\
a x^{2} & \text { if } & x=2 \\
x^{2}, & \text { if } & x>2
\end{array} \text { with } a \in[0,1]\right.
$$

Obviously, the functions $f_{1}$ and $f_{2}$ are locally Lispchitz on $X$. Now, for the function $f_{1}$ at $\bar{x}=1$ and $\alpha=1$, we have

$$
f_{1}(x)-f_{1}(\bar{x})-\left\langle A, \exp _{\bar{x}}^{-1} x\right\rangle+\alpha\left\|\exp _{\bar{x}}^{-1} x\right\|=
$$




$$
=\ln (x)-0-\ln (x)+\left|\ln \left(\frac{1}{x}\right)\right| \geq 0, \quad \forall x \in X
$$

Similarly, one can check for function $f_{2}$ at $\bar{x}=1$ and $\alpha=1$, we get

$$
\begin{gathered}
f_{2}(x)-f_{2}(\bar{x})-\left\langle B, \exp _{\bar{x}}^{-1} x\right\rangle+\alpha\left\|\exp _{\bar{x}}^{-1} x\right\|= \\
=f_{2}(x)-0-0+\left|\ln \left(\frac{1}{x}\right)\right| \geq 0, \quad \forall x \in X
\end{gathered}
$$

Therefore the functions $f_{1}$ and $f_{2}$ are AGCX and therefore AGPCX at $\bar{x}=1$ with constant $\alpha=1$ on $X$.

The point $\bar{x}=1 \in X$ is a vector critical point (VCP) since there exist some $x \in X \subseteq M$ and $\lambda=(0,1) \in \mathbb{R}_{+}^{p} \backslash\{0\}$ such that

$$
\lambda^{T}\left\langle A, \exp _{\bar{x}}^{-1} x\right\rangle=(0,1)^{T}(\ln (x), 0)=0, \text { for some } A \in \partial f(\bar{x})
$$

Further, $\bar{x}=1$ is a solution for $W S V$ problem

$$
\left\langle A, \exp _{\bar{x}}^{-1} x\right\rangle=(\ln (x), 0) \nless(0,0), \quad \forall x \in X \text { and } t \in[0,1]
$$

and $\bar{x}=1$ is a solution for WMV problem because

$$
\left\langle C, \exp _{x}^{-1} \bar{x}\right\rangle \ngtr 0, \quad \forall C \in \partial f(x)
$$

Also, for $\bar{x}=1$, we get

$$
\left(f_{1}(x)-f_{1}(\bar{x}), f_{2}(x)-f_{2}(\bar{x})\right)=\left(\ln (x)-0, f_{2}(x)-0\right) \nless(0,0), \quad \forall x \in X .
$$

Therefore, $\bar{x}=1$ is a weak efficient solution for VOP and therefore $\bar{x}=1$ is a local approximate weakly efficient (AWE) solution, the corollary 3 being verified.

\section{Application: Stackelberg Equilibrium Problem via variational problems on Hadamard manifolds}

In the same way that we can use the vector variational-like problems to reach the local approximate weakly efficient solutions of VOP, we will see that under geodesic convex functions we can reach the Stackelberg equilibrium points through variational problems on Hadamard manifolds.

The Stackelberg Equilibrium Problem (SEP) on Riemannian manifolds context is defined as

$$
\begin{array}{ll}
\text { (SEP) } & \min f_{1}\left(x_{1}, x_{2}\right) \\
& x_{1} \in K_{1} \\
& \text { s.t } \quad x_{2} \in R_{S E}=\arg \min f_{2}\left(x_{1}, x_{2}\right) \\
& \quad x_{2} \in K_{2}
\end{array}
$$

with $f_{1}, f_{2}: K_{1} \times K_{2} \rightarrow \mathbb{R}$ are the payoffs/loss functions for the players, and $K_{1}, K_{2} \subset M$ are their strategy sets on a Hadamard manifold.

The first step is to solve 


$$
\begin{aligned}
& R_{S E}\left(x_{1}\right)=\arg \min f_{2}\left(x_{1}, x_{2}\right) \\
& \quad x_{2} \in K_{2}
\end{aligned}
$$

for every fixed $x_{1} \in K_{1}$. Thus is, we must determine the optimal or best response set of the follower in SEP as a reaction function, i.e. the Stackelberg equilibrium Response set as

$$
R_{S E}=\left\{x_{2} \in K_{2}: f_{2}\left(x_{1}, y\right)-f_{2}\left(x_{1}, x_{2}\right) \geq 0, \quad \forall y \in K_{2}\right\}
$$

for every fixed $x_{1} \in K_{1}$.

The next step, for the leader player, insert the reaction function of the follower into its optimization problem, where $r$ is a reaction function selection of the set-valued map $R_{S E}$ and minimize

$$
\begin{aligned}
& S_{S E}\left(x_{1}\right)=\arg \min f_{1}\left(x_{1}, r\left(x_{1}\right)\right) \\
& \quad x_{1} \in K_{1}
\end{aligned}
$$

i.e., more precisely, the Stackelberg Equilibrium Leader set is

$$
S_{S E}=\left\{x_{1} \in K_{1}: f_{1}(x, r(x))-f_{1}\left(x_{1}, r\left(x_{1}\right)\right) \geq 0, \quad \forall x \in K_{1}\right\}
$$

An easier way to obtain those Stackelberg equilibrium points is via the solutions of variational problems.

Related to these sets we introduce two variational problems to obtain our Stackelberg equilibrium points. We define a slightly larger set than the Stackelberg equilibrium response $R_{S E}\left(x_{1}\right)$ set by means of variational inequalities.

(a) The so-called Stackelberg Variational Response set defined by

$$
R_{S V}\left(x_{1}\right)=\left\{x_{2} \in K_{2}:\left\langle\frac{\partial f_{2}}{\partial x_{2}}\left(x_{1}, x_{2}\right), \exp _{x_{2}}^{-1} y\right\rangle \geq 0, \quad \forall y \in K_{2}\right\}
$$

To check the solutions of Stackelberg variational response $R_{S V}\left(x_{1}\right)$ set it is easier than the elements of $R_{S E}\left(x_{1}\right)$, thus, we can locate the elements of the Stackelberg equilibrium response set among these points.

(b) We introduce the Stackelberg Variational Leader set

$$
S_{S V}=\left\{x_{1} \in K_{1}:\left\langle\frac{\partial f_{1}}{\partial x_{1}}\left(x_{1}, r\left(x_{1}\right)\right), \exp _{x_{1}}^{-1} y\right\rangle \geq 0, \quad \forall y \in K_{1}\right\}
$$

The set $S_{S V}$ contains the best strategies of the first player.

Let us see under what conditions sets $R_{S E}\left(x_{1}\right)$ and $R_{S V}\left(x_{1}\right)$ coincide:

Theorem 9 Let $f_{2}: K_{1} \times K_{2} \rightarrow \mathbb{R}$ be a locally Lipschitz funcion and $K_{i} \subset M$ geodesic convex sets, $i=1,2$. Then, we have the following assertions:

(a) $R_{S E}\left(x_{1}\right) \subseteq R_{S V}\left(x_{1}\right)$ for every $x_{1} \in K_{1}$.

(b) If $f_{2}\left(x_{1}, \cdot\right)$ is geodesic convex on $K_{2}$ for some $x_{1} \in K_{1} \Rightarrow R_{S E}\left(x_{1}\right)=$ $R_{S V}\left(x_{1}\right)$

Proof. 
(a) Given $x_{2} \in R_{S E}\left(x_{1}\right)$ then $f_{2}\left(x_{1}, y\right) \geq f_{2}\left(x_{1}, x_{2}\right), \forall y \in K_{2}$. By definition, one has that

$$
\left\langle\frac{\partial f_{2}}{\partial x_{2}}\left(x_{1}, x_{2}\right), v\right\rangle=\lim _{t \rightarrow 0^{+}} \frac{f_{2}\left(x_{1}, \exp _{x_{2}}(t v)\right)-f_{2}\left(x_{1}, x_{2}\right)}{t}, \quad \forall v \in T_{x_{2}} M
$$

By the geodesic convexity of $K_{2}$, then $\exp _{x_{2}}\left(t \exp _{x_{2}}^{-1} y\right) \in K_{2}$ for every $t \in[0,1]$ whenever $y \in K_{2}$. If $v=\exp _{x_{2}}^{-1} y \in T_{x_{2}} M$ in the above expression, we conclude that

$$
\left\langle\frac{\partial f_{2}}{\partial x_{2}}\left(x_{1}, x_{2}\right), \exp _{x_{2}}^{-1} y\right\rangle \geq 0
$$

which implies that $x_{2} \in R_{S V}\left(x_{1}\right)$ for every $x_{1} \in K_{1}$.

(b) We proof the reciprocal condition. By the geodesic convexity of $f_{2}\left(x_{1}, \cdot\right)$ function, we have that

$$
f_{2}\left(x_{1}, y\right)-f_{2}\left(x_{1}, x_{2}\right) \geq\left\langle\frac{\partial f_{2}}{\partial x_{2}}\left(x_{1}, x_{2}\right), \exp _{x_{2}}^{-1} y\right\rangle, y \in K_{2}
$$

Since $x_{2} \in R_{S V}\left(x_{1}\right)$, one has that

$$
\left\langle\frac{\partial f_{2}}{\partial x_{2}}\left(x_{1}, x_{2}\right), \exp _{x_{2}}^{-1} y\right\rangle \geq 0, y \in K_{2}
$$

Then $f_{2}\left(x_{1}, y\right)-f_{2}\left(x_{1}, x_{2}\right) \geq 0$ for all $y \in K_{2}$, therefore $x_{2} \in R_{S E}\left(x_{1}\right)$.

Hence, in geodesic convex context, thus is, $K_{i}, 1=1,2$ are geodesic convex sets and $f_{2}\left(x_{1}, \cdot\right)$ geodesic convex functions, we have that:

$$
R_{S E}\left(x_{1}\right)=R_{S V}\left(x_{1}\right)
$$

We have proven that the relationship between variational point and equilibrium points is obtained for geodesic convex payoff functions, extending the results obtained for convex payoff functions given by Nagy [20]. Let us illustrate this property with an example.

Example 7 Let $M=\mathbb{R}_{++}=\{x \in \mathbb{R}: x>0\}$ be endowed with the Riemannian metric defined by $g(x)=x^{-2}$.

Let $K_{1}=K_{2}=[1,2]$ be are convex sets and therefore geodesic convex sets and consider a two-player game with payoff functions defined as:

$$
\begin{gathered}
f_{1}\left(x_{1}, y\right)=x^{2}-3 x_{1}+\left|x_{1}-1.5\right| x_{2} \\
f_{2}\left(x_{1}, x_{2}\right)=\frac{x_{2}^{2}}{2}-x_{1} x_{2}
\end{gathered}
$$

We can calculate:

$$
\frac{\partial f_{1}\left(x_{1}, x_{2}\right)}{\partial x_{1}}=\left\{\begin{array}{l}
2 x_{1}-3+x_{2}, \text { if } x_{1}>1.5 \\
2 x_{1}-3-x_{2}, \text { if } x_{1}<1.5
\end{array}\right.
$$




$$
\frac{\partial f_{2}\left(x_{1}, x_{2}\right)}{\partial x_{2}}=x_{2}-x_{1}
$$

The $f_{2}\left(x_{1}, \cdot\right)$ is geodesic convex on $K_{2}$ for every $x_{1} \in K_{1}$, then for previous Theorem 9, the Stackelberg Variational Response is

$$
\begin{aligned}
R_{S V}\left(x_{1}\right)= & \left\{x_{2} \in K_{2}:\left\langle\frac{\partial f_{2}}{\partial x_{2}}\left(x_{1}, x_{2}\right), \exp _{x_{2}}^{-1} y\right\rangle \geq 0, \quad \forall y \in K_{2}\right\}= \\
& =\left\{x_{2} \in K_{2}: x_{2}=x_{1}, x_{1} \in K_{1}\right\}=R_{S E}\left(x_{1}\right)
\end{aligned}
$$

and in this case, the Stackelberg Variational Leader set and Stackelberg Equilibrium Response are

$$
\begin{gathered}
S_{S V}=\left\{x_{1} \in K_{1}:\left\langle\frac{\partial f_{1}}{\partial x_{1}}\left(x_{1}, r\left(x_{1}\right)\right), \exp _{x_{1}}^{-1} y\right\rangle \geq 0, \quad \forall y \in K_{1}\right\} \\
S_{S V}=\{(1,1)\}=S_{S E}\left(x_{1}\right)
\end{gathered}
$$

In our case, this solution is the point $(\bar{x}, \bar{y})=(1,1)$, which is both a Nash Equilibrium point.

\section{Conclusions}

This paper, for the first time, we have introduced concepts such as local approximate weakly solutions or generalized approximate geodesic convex functions, to demonstrate that we can achieve local approximate weakly solutions by using Stampacchia and Minty type solutions on Hadamard manifolds. We can also obtain Stackelberg equilibrium points via solutions to variational problems in the case of geodesic convex payoff functions, improving on previous results in the literature. The results presented in this paper lead to the following conclusions:

- We have extended the approximate and weakly approximate solutions given by Mishra and Upadhyay [19] and Wang et al. [28] in linear spaces to Hadamard manifolds.

- Inspired by the work of Ngai, Luc and Thera [21], we have introduced the concepts of generalized approximate geodesic convex functions on Hadamard manifolds, and we have illustrated them with examples.

- We have proved that, under conditions of approximate geodesic convexity functions, the solutions of the Stampacchia Vector Variational-Like Inequality Problem are local approximate efficient points $(A E)$.

- We have shown the relationships between the local approximate efficient solutions for $V O P$ and the solutions of Minty Vector Variational-Like Inequality Problem $(M V)$.

- We have been able to identify solutions to Stampacchia Weak Vector Variational-Like Inequality Problem $(S W)$ with the local approximate weakly efficient $(A W E)$ points for VOP. 
- We have gone one step further, and we have proved that approximate geodesic pseudoconvexity (resp. approximate geodesic strictly pseudoconvexity) is a minimal requirement for the property that every VCP is a local weakly approximate efficient (resp. approximate efficient) solution for VOP on a Hadamard manifold in the non-smooth case.

- Under approximate geodesic pseudoconvex conditions, we have identified Vector Critical Points, approximate weakly efficient points for VOP and solutions of Weak Stamppacchia and Minty Variational-like inequalities problems just as we proved with finite-dimensional Euclidean spaces.

- We have introduced two variational problems to obtain Stackelberg equilibrium points, and we have proved under what conditions the points of these sets coincide.

In our opinion, in the future, algorithms should be designed to obtain approximate efficient solutions from a non-smooth vector optimization problem and look for more economical applications of the theoretical results achieved here.

\section{References}

1. Q.H. Ansari, M. Islam and J.C. Yao, Nonsmooth variational inequalities on Hadamard manifolds, Applicable Analysis, 99 (2020) 340-358. DOI: 10.1080/00036811.2018.1495329.

2. S.I. Chen and C.J. Fang, Vector variational inequality with pseudoconvexity on Hadamard manifolds, Optimization 65, (2016), 2067-2080. DOI: 10.1080/02331934.2016.1235161.

3. S.I. Chen and N.J. Huang, Vector variational inequalities and vector optimization problems on Hadamard manifolds, Optim. Lett., 10 (2016), 753-767. DOI: 10.1007/s11590015-0896-1.

4. S. I. Chen, N. J. Huang and D. O'Regan, Geodesic B-preinvex Functions and Multiobjective Optimization Problems on Riemannian Manifolds, Journal of Applied Mathematics, (2014), Article ID 524698, DOI: 10.1155/2014/524698.

5. V. Colao, G. López, G. Marino and V. Martín-Márquez, Equilibrium problems in Hadamard manifolds, J. Math. Anal. Appl. 38 (8) (2012), 61-77. DOI: 10.1016/j.jmaa.2011.11.001.

6. P.T. Fletcher, S. Venkatasubramanian and S. Joshi, The geometric median on Riemannian manifolds with application to robust atlas estimation, Neuroimage 45 (2009), 5143-5152. DOI: 10.1016/j.neuroimage.2008.10.052.

7. M. Gabarrou, D. Alazard and D. Noll, Design of a flight control architecture using a non-convex bundle method, Math. Control Signals Syst, 25 (2013), 257-290. DOI: 10.1007/s00498-012-0093-z.

8. F. Giannessi, Theorems of alternative quadratic programs and complementarity problems. In: Cottle R.W., Giannessi F. Lions J.L., editors. Variational inequalities and complementarity problems. Theory and Applications. New York, Wiley, (1980), 151186.

9. F. Giannessi, On Minty variational principle. New trends in mathematical programmig. Boston. Kluwer Academic Publishers (1998), 93-99.

10. P. Grohs and S. Hosseini, $\epsilon$-subgradient algorithms for locally lipschitz functions on Riemannian manifolds, Adv. Comput. Math., 42 (2016), 333-360. DOI: 10.1007/s10444015-9426-z.

11. C. Gutiérrez, R. López and V. Novo, Generalized $\epsilon$-quasi-solutions in multiobjective optimization problems: existence results and optimality conditions, Nonlinear Analysis 72 (2010), 4331-4346. 
12. A. Jayswal, I. Ahmad and B. Kumari, Vector variational inequalities on Hadamard manifolds involving strongly geodesic convex functions, Operations Research Letters 47 (2019), 110-114. DOI: 10.1016/j.orl.2019.01.004.

13. A. Jofré, D.T. Luc and M. Thera, $\epsilon$-subdifferential and $\epsilon$-monotonicity. Nonlinear Analysis, 33 (1998), 71-90.

14. A. Kristály, Nash-type equilibria on Riemannian manifolds: A variational approach, Journal de Mathématiques Pures et Appliquées, 101 (5) (2014), 660-688. DOI: 10.1016/j.matpur.2013.10.002.

15. S.S. Kutateladze, Convex $\epsilon$-programming, Sov Mathe Dokl, 20 (1979), 391-393.

16. H. Li, D. Chen, H. Zhang, C. Wu and X. Wang, Hamiltonian analysis of a hydro-energy generation system in the trasient of sudden local increasing, Applied Energy 185 (2017), 244-253. DOI: $10.1016 /$ j.apenergy.2016.10.080.

17. Y. Ma, J. Kosecka and S.S. Sastry, Optimization criteria and geometric algorithms for motion and structure estimation, Int. J. Computer Vision, 44 (3) (2001), 219-249. DOI: 10.1023/A:1012276232049.

18. Y. Nishimori and S. Akaho, Learning algorithms utilizing quasi-geodesic flows on the Stiefel manifold, Neurocomputing 67 (2005), 106-135. DOI: 10.1016/j.neucom.2004.11.035.

19. S.K. Mishra and B.B. Upadhyay, Some relations between vector variational inequality problems and nonsmooth vector optimization problems using quasi efficiency, Positivity, 17 (2013), 1071-1083. DOI: 10.1007/s11117-013-0221-0.

20. S. Nagy, Stackelberg equilibria via variational inequalities and projections, J. Glob. Optim., 57 (2013), 821-828. DOI: 10.1007/s10898-012-9971-7.

21. H.V. Ngai, D.T. Luc and M. Thera, Approximate convex functions. J. Nonlinear Convex Anal., 1 (2000), 155-176.

22. A.J. Novak, G. Feichtinger and G. G. Leitmann, A differential game related to terrorism: Nash and Stackelberg strategies. J. Optim. Theory Appl. 144 (2010), 533-555.

23. G. Ruiz-Garzón, R. Osuna-Gómez, A. Rufián-Lizana, Relationships between vector variational-like inequality and optimization problems. European J. Oper. Res. 157, (2004), 113-119. DOI: 10.1016/S0377-2217(03)00210-8.

24. G. Ruiz-Garzón, R. Osuna-Gómez, A. Rufián-Lizana, and B. Hernández-Jiménez, Optimality and Duality on Riemannian Manifolds. Taiwanese Journal of Mathematics, 22 (5) (2018), 1245-1259. DOI: $10.11650 / \mathrm{tjm} / 180501$.

25. G. Ruiz-Garzón, R. Osuna-Gómez and J. Ruiz-Zapatero, Necessary and Sufficient Optimality Conditions for Vector Equilibrium Problems on Hadamard Manifolds. Symmetry, 11 (2019), 1037; DOI:10.3390/sym11081037.

26. G. Ruiz-Garzón, R. Osuna-Gómez and A. Rufián-Lizana, Solutions of Optimization Problems with Lipschitz Functions. Symmetry, 12 (2020), 804; DOI:10.3390/sym12050804.

27. H. von Stackelberg, Marktform und Gleichgewicht, Berlin, Verlag von Julius Springer, (1934).

28. Z. Wang, R. Li and G. Yu, Vector critical points and generalized quasi-effciient solutions in nonsmooth multi-objective programming, Journal of Inequalities and Applications, 184 (2017), DOI: 10.1186/s13660-017-1456-2.

29. X. Wang, G. López, C. Li and J.C. Yao, Equilibrium problems on Riemannian manifolds with applications, J. Math. Anal. Appl. 473 (2019), 866-891. DOI: 10.1016/j.jmaa.2018.12.073 